\title{
FRONTIERS OF
}

CHARACTERIZATION

AND METROLOGY FOR

NANOELECTRONICS

2009 International Conference on Frontiers of

Characterization and Metrology for Nanoelectronics

Albany, New York $\quad 11-15$ May 2009

EDITORS

David G. Seiler

National Institute of Standards \& Technology

Gaithersburg, Maryland

Alain C. Diebold

College of Nanoscale Science \& Engineering SUNY Albany, New York

All papers have been peer-reviewed.

SPONSORING ORGANIZATIONS

National Institute of Standards \& Technology

College of Nanoscale Science \& Engineering, SUNY Albany

Semiconductor Equipment \& Materials International

Semiconductor Research Corporation

International SEMATECH Manufacturing Initiative

Semiconductor International

National Science Foundation

American Physical Society

American Vacuum Society

\section{EXHIBITORS}

Bruker AXS, Inc.

Omicron NanoTechnoloy, USA

CD-ROM INCLUDED
Robert McDonald

Technology Associates

Monte Sereno, California

C. Michael Garner

Intel, Santa Clara, California

Dan Herr

Semiconductor Research Corporation Research Triangle Park, North Carolina

Rajinder P. Khosla National Science Foundation Arlington, Virginia

Erik M. Secula National Institute of Standards \& Technology Gaithersburg, Maryland

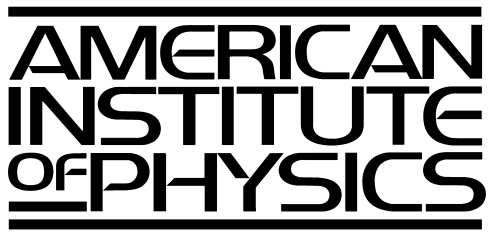




\title{
Characterization of Integrated Nano Materials
}

\author{
Amal Chabli, Peter Cherns, Nicolas Chevalier, David Cooper, Dominique \\ Lafond, François Bertin, Henri Blanc, Ariel Brenac, Philippe Andreucci \\ and Jean-Christophe Gabriel
}

CEA, LETI, MINATEC, F38054 Grenoble, France

\begin{abstract}
Depending on the level of the technological developments, the characterization techniques are mature to support them or still require protocol definition and relevance demonstration for the issues addressed. For Beyond CMOS and Extreme CMOS devices, the integration of nano-objects like nanowires and carbon nanotubes, brings about analysis requirements that are at the frontier of the state-of-the-art characterization techniques. The specific limitations of the use of the existing physical and chemical characterization techniques for integrated nanomaterials are highlighted. In the case of Scanning Probe Microscopy, in-situ localization and positioning are specifically challenging and data analysis is mainly statistical. It is also shown how specific sample preparation may serve the extraction of the required 3D information in particular for Electron Microscopy. The measurement developments related to NEMS technologies guided by the need for dynamic characterization of these components are covered too.
\end{abstract}

Keywords: 3D characterization, Microscopy, Tomography, Dynamic properties, Nanowires, Nanotubes, Nanoneedle. PACS: 68.37.-d; 81.07.-b

\section{INTRODUCTION}

The increasing interest for size effects on material properties has driven an important effort in the development of physical and chemical characterization techniques at the nanoscale. Impressive capabilities in terms of sensitivity, selectivity, depth resolution and spatial resolution are demonstrated. They may be based on huge instrumental effort including synchrotron radiation use [1] or advanced configurations of probe-sample interaction such as the inelastic electron tunneling spectroscopy performed in a scanning tunneling microscope [2]. Moreover, powerful simulations for data interpretation are required to extend for example the use of $\mathrm{x}$-ray to the structural characterization of nanocrystalline materials [3]. All these improvements sustain nanoscience research promoting innovative nanocomponent design.

However, only few characterization developments address the integration issues. The integration of nano material has to deal with process selectivity; process reproducibility and uniformity; self organisation; localisation; connection and manipulation at the nanoscale while keeping the basic properties of the nanomaterial during these processes. The integration of nano-objects like nanowires and carbon nanotubes, brings about analysis requirements that are at the frontier of the state-of-the-art characterization techniques. Furthermore, the Beyond CMOS technology issues would call for anticipating the characterization challenges related to the 3Dinformation that will be needed.

In this paper, we highlight the specific limitations of the use of the existing physical and chemical characterization techniques for integrated nanomaterials. Extension of the 2D information capabilities of Scanning Probe Microscopy for the characterization of integrated nanostructures will be overviewed. Then we show how specific sample preparation may serve the extraction of the required 3D information, taking electron microscopy as a demonstration technique. Finally, the 3D integration and NEMS (Nano Electro Mechanical Systems) technologies set specific characterization conditions related to the very high aspect ratio and to the motion of the integrated nanostructures that will be pointed out.

\section{CHARACTERIZATION CHALLENGES VS ISSUES OF BEYOND CMOS AND MEMS/NEMS TECHNOLOGIES}

It is commonly considered that the technological research is governed by three main driving forces. The

CP1173, Frontiers of Characterization and Metrology for Nanoelectronics: 2009

edited by D. G. Seiler, A. C. Diebold, R. M cD onald, C. M. Garner, D. Herr, R. P. K hosla, and E. M. Secula (c) 2009 A merican Institute of Physics 978-0-7354-0712-1/09/\$25.00 
micro and nanotechnology driving forces are depicted schematically on figure 1 regarding both the device scaling down level and the technological node considered. The first driving force is the continuous scaling down requirements of the "More Moore" developments. The second one is the complexity increase of the device functions with the use of other material properties than the electronic ones (mechanical, thermal, piezoelectric, biochemical, optical...) leading to the "More Than Moore" developments. The convergence of this two driving force is expected to result from the 3D integration below the $22 \mathrm{~nm}$ node. The third driving force, related to the so called "beyond CMOS" developments, is a bottom up approach. While the two first driving forces are used to deal with integration of materials, the last one is still at the stage of understanding and controlling the physical and chemical properties of different kinds of nanoscale materials.

Even though, the technological investigations are always concerned by new material introduction and new integration process evaluation in order to meet the requirements of these driving forces. The role of the physical and chemical characterization is to support all together, the material choice and its improvement, the analysis of scaling effects and their use or correction, and the design of integration processes and their validation. Depending on the level of the technological developments, the characterization techniques are mature to support them or still require protocol definition and relevance demonstration for the issues addressed.

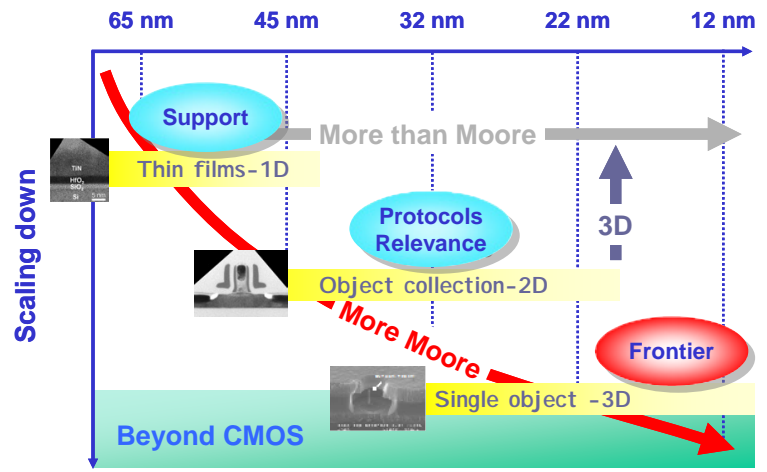

FIGURE 1. Status of the physical and chemical characterization developments as required by the micro and nanotechnology driving forces.

As indicated in figure 1, until the $45 \mathrm{~nm}$ node, the integrated materials are in general available as full wafer layers. The process improvement and control requires mainly 1D information. However, the subnanometer depth resolution is mandatory due to the layer thickness decrease with device scaling down.
During the ten last years, several characterization techniques have been demonstrated to reach the subnanometer depth resolution in specific configurations for the analysis of metal gate stacks or $\mathrm{Cu}$ interconnect stacks. At least four of them may be mentioned: (i) Secondary Ion Mass Spectrometry (SIMS) using Cs primary ions and MCs secondary cluster ions for the $M$ atom analysis [4], (ii) Medium Energy Ion Scattering (MEIS) with its specific sensitivity to heavy atoms like $\mathrm{Hf}$ or $\mathrm{Zr}$ [5], (iii) Photoelectron Spectroscopy (PES) using the angle resolved mode for the selectivity of the analysis depth [6,7] and (iv) infrared spectrometry in the Attenuated Total Reflection optical configuration (ATR) [8]. They are always complementary to electron microscopy even if this latter technique shows the ultimate spatial resolution in the transmission mode (TEM). Indeed, TEM is still limited by its poor chemical sensitivity and both the time consuming and the artifact generation of the sample preparation.

Below the $45 \mathrm{~nm}$ node, several integrated materials are not available on full wafers and process steps simulated on full wafers are no more representative of the integration processes. This is the case of the SiGe selective epitaxy, growth of self aligned barriers, narrow $\mathrm{Cu}$ lines in damascene structures and ultra shallow doping of source and drain areas. Then, the support of the technology development requires mainly 2D information. This has led to an impressive improvement of the characterization techniques in terms of lateral resolution inducing the set up of imaging capabilities too. Indeed, SIMS in the TimeOf-Flight mass selection mode together with the dual primary source shows imaging capabilities that allow $\mathrm{Cl}$ contamination analysis in $\mathrm{Cu}$ lines [9]. Thanks to the introduction of aberration corrected electron analyzers, full field imaging PES demonstrates highly promising spatial resolution for chemical surface analysis [10]. Also, electrical Scanning Probe Microscopies (SPMs), based on Atomic Force Microscopy (AFM), gained specific attention related to the various operating modes that allow selecting the probe-sample interaction depending on the wanted information. Thus, Scanning Probe Microscopy (SCM) and Scanning Spreading Resistance Microscopy (SSRM) are used in contact mode with a conducting probe for doping imaging and junction delineation capabilities [11-15]. More recently, local work function measurement by Kelvin Force Microscopy (KFM) has been proposed to support the metal gate developments [16]. Generally, surface topography is a limiting parameter for reliable image interpretation.

At the same time, a great effort has been undergone to improve TEM column stability and aberration correction allowing uppermost spatial resolution [17] and increased sensitivity through longer integration 
times. This is highlighted in figure 2 that shows the effect of the increased integration time on the signal to noise ratio in phase images obtained on a CMOS device examined using off-axis electron holography.

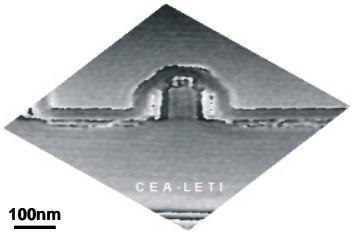

(a)

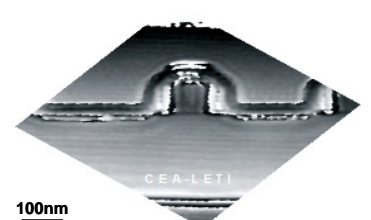

(b)
FIGURE 2. Reconstructed phase image obtained using offaxis electron holography on a CMOS device with an integration time of (a) $4 \mathrm{~s}$ and (b) $45 \mathrm{~s}$ thanks to the column stability of the FEI Titan microscope and a well controlled TEM environment.

Holograms recorded for integration times up to $128 \mathrm{~s}$ result in measuring steps in potential of less than $0.030 \pm 0.003 \mathrm{~V}$ giving access to an improved sensitivity in 2D doping profiles as shown on figure 3. For that, the TEM specimens are prepared as lamellae that need to be in the thickness range 400 to $500 \mathrm{~nm}$ that should be as constant as possible on the whole field of view [18].

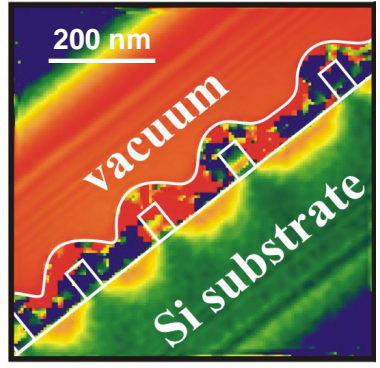

FIGURE 3. Reconstructed phase image in false colors obtained using off-axis electron holography on $45 \mathrm{~nm}$ CMOS structures thanks to a controlled sample thickness on a wide field of view and the TEM stability.

Nevertheless, one has to take sufficient care during sample preparation to obtain quantitative information as reported by D. Cooper et al. [19]. In addition, the reliable analysis is based on the assumption of constant properties throughout the whole specimen thickness.

Despite these effective and decisive improvements in sensitivity, selectivity and spatial resolution, the 45 $\mathrm{nm}$ node technology developments are still waiting for some relevance demonstration of the analysis techniques and the set-up of specific characterization protocols.

Then, below the $22 \mathrm{~nm}$ node, the 3D integration and the integration of nanomaterials is bringing analysis requirements that are at the frontier of the state-of-the-art characterization techniques. Furthermore, the Beyond CMOS technology issues would call for anticipating specific characterization challenges. For example, the presence of a nanoscale material within the lamella specimen for TEM is no more respectful of the constant properties assumption in the lamella thickness. Thus, a 3D-information is mandatory to overcome the breakdown of this assumption.

As a matter of fact, ongoing comprehensive developments have to provide the $3 \mathrm{D}$ information that will be required. We will not elaborate on the whole developments covering $3 \mathrm{D}$ characterization. An extensive literature may be found in a very recent review paper [20]. TEM tomography [20], X-ray tomography [21] or atom probe analysis [22,23] are clearly of interest in our field. Instead we will focus on the possible extension of $2 \mathrm{D}$ characterization to integrated nanomaterials and on the TEM capabilities for tomography. This will highlight the mandatory specific sample preparation developments to get reliable 3D information from a general point of view. Then a particular attention will be given to the characterization issues related to devices based on nanomaterial motion like NEMS.

\section{D ELECTRICAL SPM CHARACTERIZATION}

As indicated previously, electrical SPMs are widely used to probe samples with low surface topography. They are also easily applied to study transport properties of individual nanomaterials using specific contact design and nanomaterial positioning [24]. However, it is not straightforward to extend their use to integrated nanomaterials like vertically grown nanotubes and nanowires (NWs) on a substrate. Figure 4 shows a scheme of the possible extension of the use of the electrical SPMs to characterize the electrical properties of as grown vertical nanomaterials.

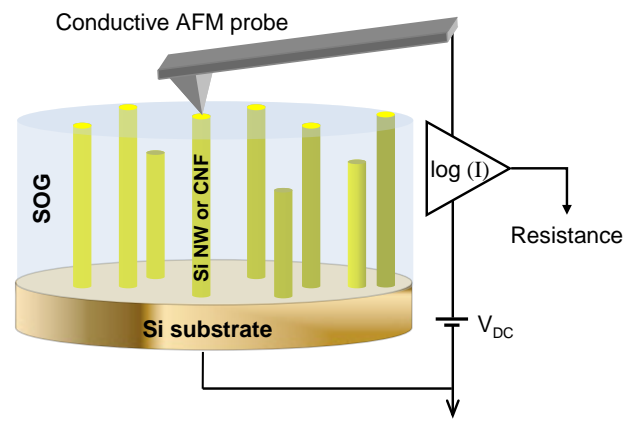

) ,* 85( $1 \square$ Scheme of the electrical Electrical SPM characterization of as grown vertical nanomaterials SSRM configuration used for local electrical characterization 
They may be first embedded in a dielectric material like a polymer or a spin on glass (SOG) as shown by the Scanning Electron Microscopy (SEM) cross section of carbon nanofibers (CNF) in figure 5.

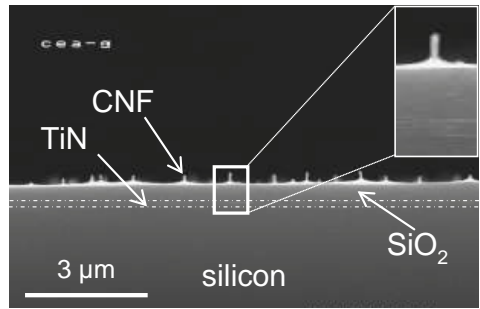

FIGURE 5. SEM view of CNF embedded in SOG to allow SPM electrical measurements.

SSRM measurements performed on these embedded CNF grown on a TiN layer are given in figure 6 . A one to one correlation between topography and electrical images indicates that bright spots on the SSRM images are related to individual CNF.

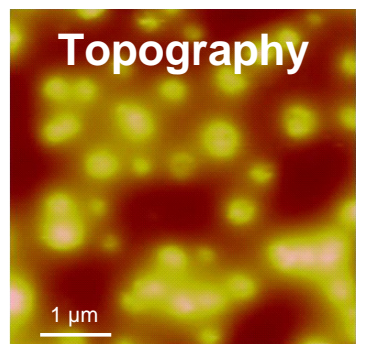

(a)

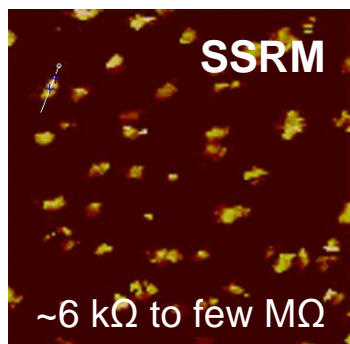

(b)
FIGURE 6. SPMs in contact mode on embedded vertical CNF: (a) AFM topography (amplitude range of about 300 $\mathrm{nm}$ ), (b) SSRM measurement using a PtIr coated silicon tip (brightest points in the electrical image correspond to the more conductive CNF).

A resistance distribution is observed and is widely discussed by L. Fourdrinier et al. [25]. Apart from the explanation for the measured resistance distribution which is assumed to be related to the TiN layer roughness, these measurements shows that the properties of the integrated nanomaterials would highly depend on their environment and they would hardly keep their intrinsic properties. At least, only a statistical analysis as given in figure 7 is significant considering these integrated CNF.

In the same manner, SPM characterizations were performed on non-intentionally doped n-type $\mathrm{ZnO}$ nanowires embedded in a polymer matrix. Transport properties investigations using SCM revealed local ptype space charge regions spreading over the outer shell of the polymer-coated $\mathrm{ZnO} \mathrm{NW}$. Correlatively, different electrical behaviors were found for the core and shell parts of the nanowires using SSRM.

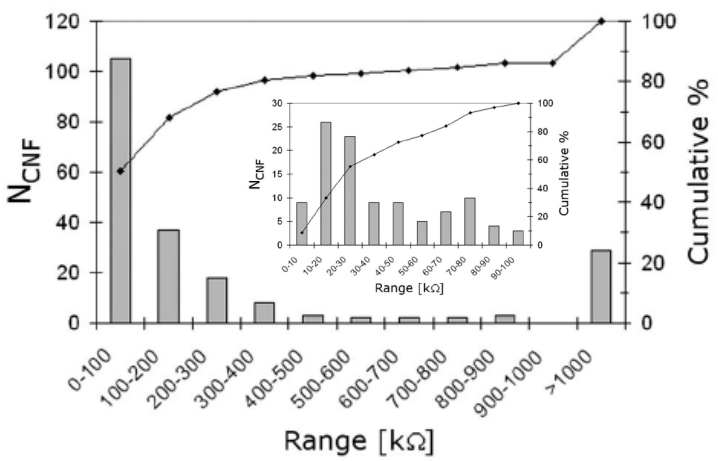

FIGURE 7. SPMs in contact mode on embedded vertical CNF: statistical analysis of the SSRM measurement performed on about 200 CNFs after calibration and conversion of the SSRM signal in resistance units [26].

Examples of the results are shown on figure 8. A self-consistent electrical transport model was developed based on Fermi level pinning at surface states arising from the surrounding environment [27]. Here again, the 2D electrical measurements have shown that keeping the intrinsic properties of nanomaterials during their integration is a central issue.

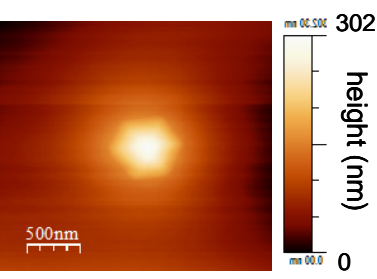

(a)

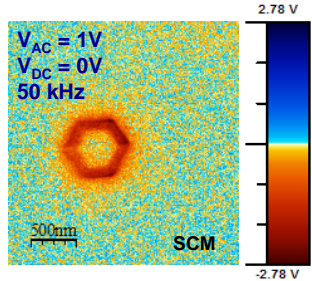

(b)
FIGURE 8. ZnO nanowire embedded in polymer: (a) AFM topography; (b) SCM measurement using a PtIr coated silicon tip.

Through these experiments, SPMs are revealed as powerful techniques for the analysis of vertically integrated nanomaterials with almost no sample preparation. However, their extensive use is still limited by AFM tip wearing that is not easily detected during the measurements. Only a post-measurement inspection under SEM observation helps to control the tip state as illustrated in figure 9 .

Moreover, in the reported experiments, both CNFs and $\mathrm{ZnO} \mathrm{NWs}$ are randomly grown on the wafer. Thus, the AFM probe does not need to be accurately positioned on the sample and the electrical path is easily defined by substrate back contact (fig. 4).

Then SPM measurements become challenging when in-situ localization and positioning are required at the nanoscale. On one hand, the magnification of the optical microscopes available on the commercial AFM 
tools is not always sufficient to allow direct localization of the feature to be analyzed. Time consuming successive SPM imaging must be performed before the final acquisition. This is not compatible with tip life time issues. On the other hand, depending on the nanomaterial integration architecture, the electrical path for the SPM measurements is not possible through the substrate back contact.

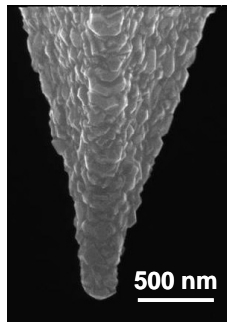

(a)

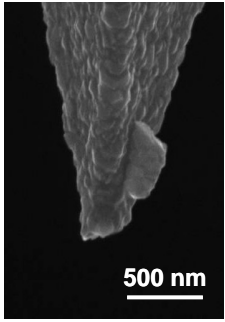

(b)

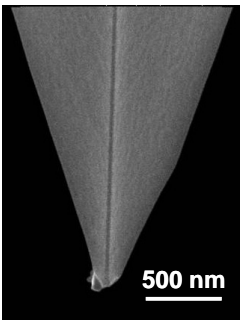

(c)
FIGURE 9. SEM observation of SPM tip: (a) Diamond coated Si tip before use; (b) Diamond coated Si tip and (c) PtIr coated Si tip after SSRM measurements.

To overcome these limitations, coupling SEM with AFM has been previously proposed. Electrical contact issues are specifically addressed by Troyon et al.[28]. Another emerging solution is a multiprobe system as proposed by X. Lin et al. [29].

\section{D TEM CHARACTERIZATION}

As pointed out above, when the sample structure is not homogeneous through the whole TEM lamella thickness, the 3D information becomes mandatory for a complete analysis. The device shown on figure 10 exemplifies clearly that kind of configuration.

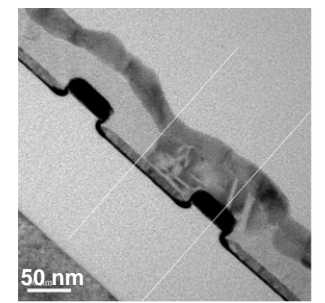

(a)

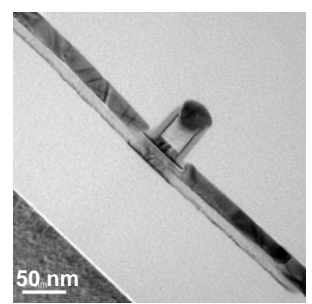

(b)
FIGURE 10. 2D images bright field TEM of a complex device inducing a TEM sample lamellae with in-depth heterogeneous structure: (a) lamella A and (b) lamella B (lamellae $\mathrm{A}$ and $\mathrm{B}$ are perpendicular to each other, lines added to the bright field image of lamella $\mathrm{A}$ indicate the position of the lamella B).

Two separated TEM lamellae has been prepared to control the morphology of the devices from perpendicular observations as shown on figures 10a and b. Different devices have been used to get these two observations making difficult to extract correlative information. Figure 11 shows the 2D high angle annular dark field (HAADF) image obtained on a TEM lamella prepared as indicated by the lines added on the bright field image of figure 10a. As a matter of fact, the full structure of the device may not be identified from the unique view of this complex device.

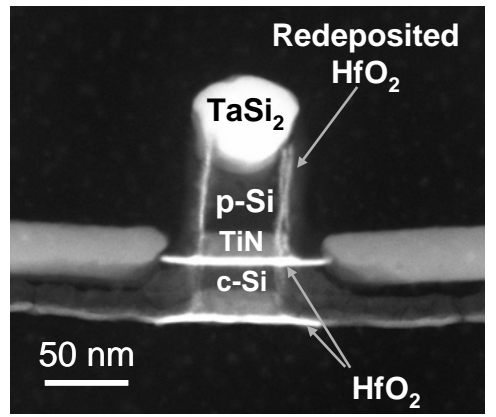

FIGURE 11. HAADF STEM image of lamella B of fig. 10.

High-resolution 3D tomography imaging in TEM may be obtained when images are recorded every one or two degrees about a tilt axis, over a specimen tilt range as large as possible. These series of tilted images is 'back-projected' to form a full 3-dimensional image of the sample, namely the 3D reconstruction. In principle, all the imaging modes available in the TEM may be used.

The powerful 3D information has been previously reported for quantitative analysis of pore size in low $\mathrm{k}$ materials [30]. Taking advantage of the distinctly different plasmon loss for $\mathrm{Si}$ and $\mathrm{SiO}_{\mathrm{x}}$, the complicated shapes of nanosized particles of silicon embedded in silicon oxide has been demonstrated using 3D reconstruction from TEM tilt series [31]. They were previously assumed as simple spheres. This emphasizes the limited information provided by conventional 2-dimensional electron-microscope images for this kind of nanomaterials.

For the device of figure 10, the lamella B has been used for analysis by electron tomography on a probe corrected Titan TEM from FEI. To allow high tilt for tomography, a Fischione 2020 sample holder is used. All tilt series have been acquired using HAADF STEM imaging. Each tilt series consists of 151 images, acquired with $1^{\circ}$ steps, between $\pm 75^{\circ}$. The post-acquisition tilt series alignment and 3D reconstruction was carried out using the FEI Inspect 3D software. The simultaneous iterative reconstruction technique (SIRT) algorithm, with 25 iterations, was used for the reconstruction. Visualisation and analysis has been carried out using the visualisation software suites Amira and Chimera. The reconstructed three- 
dimensional image is shown on figure 12 and the 3D structure of the device is revealed. This structure could not be resolved with the conventional 2D TEM observation.

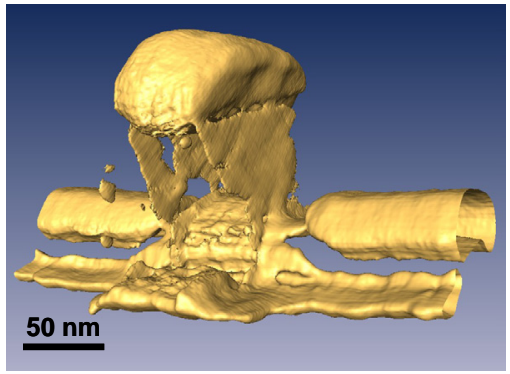

FIGURE 12. Reconstructed $3 D$ image from HAADF STEM tilt series of the device of figure 10 .

However, even with a 3D reconstruction, an accurate analysis of the results may reveal some artifacts that will be highlighted on the next example. Figure 13a shows the sample structure under concern which is based on NWs integration through a top down process described elsewhere [32]. First tilt series are recorded on a conventional lamella (fig. 13b) obtained by Focused Ion Beam (FIB) sample preparation.

(a)

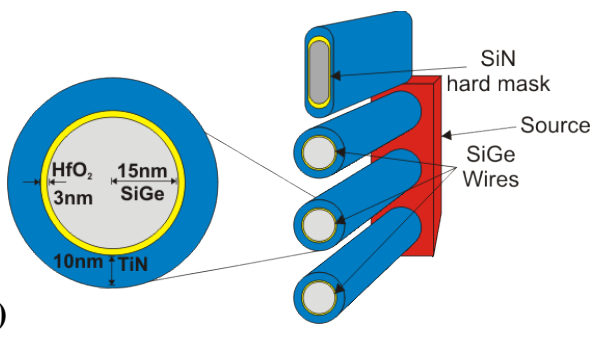

(b)

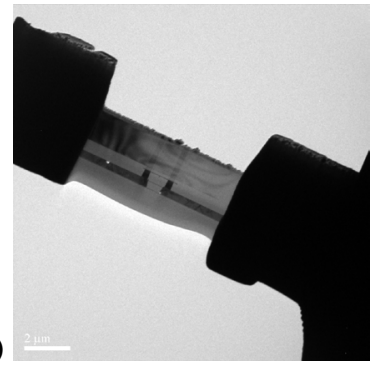

FIGURE 13. NWs device TEM tomography imaging (a) Nanowire schematic structure; (b) SEM view of the lamella sample preparation.

As demonstrated by the two selected views of the tilt series in figure 14, the wire cross section is lost during the reconstruction. Indeed, at the high tilt angle, the sample thickness causes degradation in image quality and shadowing from heavy elements. Both these problems limited the available tilt range and therefore increased distortions caused by the missing wedge.

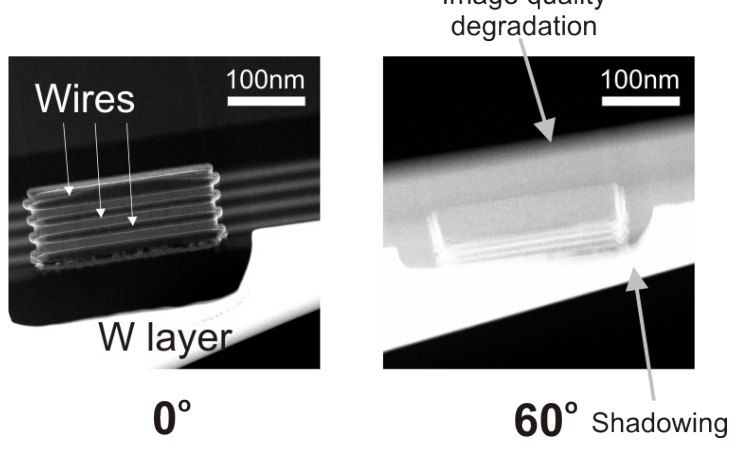

FIGURE 14. NWs device TEM tomography imaging: Two views of the TEM image series acquired for tomography.

To overcome these limitations, use of needle shaped specimens prepared using annular milling in the FIB has been previously proposed [33, 34]. The end result is that the device is isolated inside a $350 \mathrm{~nm}$ diameter needle (fig. 15a and b). The preparation in this case is more specialized and therefore more timeconsuming, but the final sample can be tilted over the full tilt range of the holder without issues of shadowing or thickness changes, as demonstrated in figure 15c.

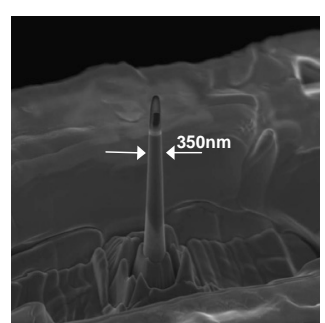

(a)

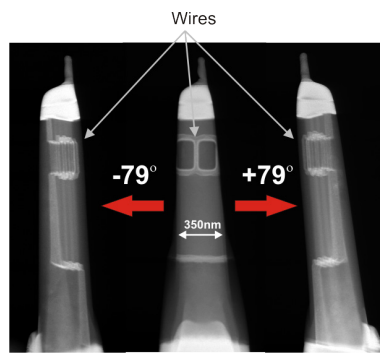

(c)

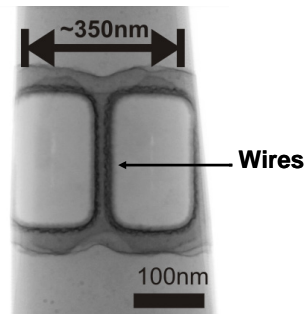

(b)

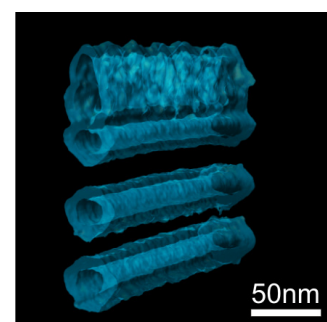

(d)
FIGURE 15. Needle shaped specimen for reliable 3D TEM tomography on integrated NWs: (a) SEM view of the FIB prepared needle; (b) HAADF STEM image showing the vertically aligned NWs in the needle; (c) extreme angle images of the tilt series (d) Iso-surface representation of NWs device showing boundaries of TiN layers. 
Figures 15d demonstrate that the reconstruction from the NWs device has been successful. An isosurface representation of the tomogram is presented. The threshold in this image is set at the intensity level of the TiN encapsulating layer. In this case, the SiGe wire is not directly represented, but the location of its boundary is inferred from the shape of the $3 \mathrm{~nm} \mathrm{HfO}_{2}$ surround, allowing analysis of changes in thickness and morphology on the whole length of the NWs.

As a summary of this section, 3D information clearly appears as mandatory for the characterization of integrated nanomaterials. Moreover, we have made a specific focus on the sample preparation issue even if it is not the only one. Even with the optimized needle preparation, depending on the sample in-depth structure, some reconstruction artefacts may still occur and will be analysed elsewhere. This call for the development of improved reconstruction algorithms that should take into account complementary information obtained from chemical analysis for example [35].

\section{NANOSCALE DYNAMICAL CHARACTERIZATION}

NEMS devices are emerging as a new field of interest because they can simultaneously offer compatibility with CMOS integration and also specific advantages such as the potential for ultra-miniature size elements, low power consumption, high resonant frequencies and enhanced detection sensitivity. NEMS can be used in a wide range of applications such as low-pass sensors (accelerometer, etc.), resonant sensors (mass sensor, etc.), or even clock time references [36]. Thus integrating on the same die a CMOS circuit and a NEMS is of increasing interest from the technological point of view. This integration brings about specific characterization challenges related to the NEMS structures that show unusual aspect ratios with regards to the CMOS technologies. Indeed, structures like the one viewed on figure 16 associate micrometer scale together with nanometer scale dimensions, implying a high spatial resolution together with a wide field of view for the morphological characterization.

Since NEMS are aimed to be used for various applications like chemical sensors, not only morphological characterizations are needed but also the use of all the powerful chemical and electrical characterization techniques mentioned in the previous sections is required. It is obvious that these NEMS structures will call for some specific sample preparations that are not covered here.

In addition to the challenges related to the characterization of integrated nanomaterials, the
NEMS manufacturing process faces a specific issue related to the control of the dynamical performances of the mobile parts of the devices.

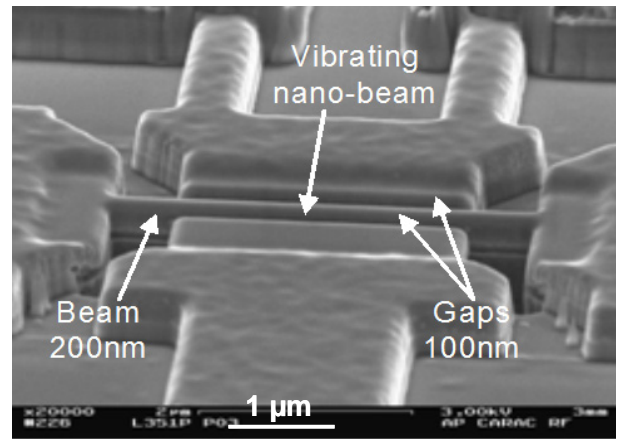

FIGURE 16. SEM view of a clock time reference radio frequency NEMS with high aspect ratio features.

Up to now, one has to wait for the complete manufacturing process, including the encapsulation step, to test individual devices. Figure 17 shows the results of the mechanical device test through the final capacitive detection that requires interconnect level integration.

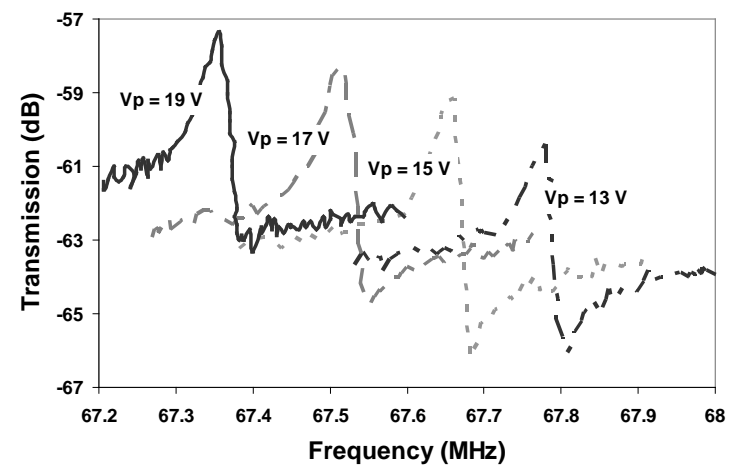

FIGURE 17. Dynamical characterization using capacitive detection of the clock time reference radio frequency NEMS of figure 16 .

In order to improve the throughput of the electromechanical devices production, it would be desirable to characterize the dynamic behavior of the mechanical elements at earlier stages of integration, specifically before some interconnection integration steps. However, while many methods have been dedicated for the mechanical assessment of thin films, only few are able to address micromechanical devices [37]. Among them, optical stroboscopic interferometry has been reported for dynamic characterization of nanostructures for both out of plane and in plane vibrations [38]. This technique has proved its efficiency for MEMS, but its extension to NEMS faces a spatial resolution limitation. More recently, 
improvements in spatial resolution are proposed by using electron stroboscopic detection [39]. The motion is detected by scanning the SEM electron beam across the edge of the mobile structure and analyzing the yield of the secondary electrons. This characterization still requires integrated actuation of the NEMS and does not overcome interconnect process step for the NEMS test.

In a very large scale integration (VLSI) scheme for NEMS manufacturing, the measurement of dynamical properties at intermediate process steps, before device completion, is the key for effective process improvements and manufacturing cost reduction. Indeed, it is foreseen that a large number of mobile structures will be present on the same wafer as shown on figure 18.

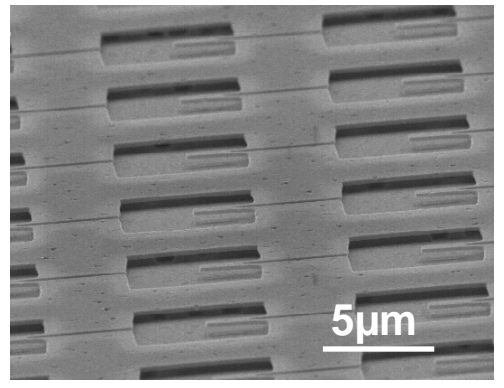

FIGURE 18. SEM view of NEMS array manufactured in a VLSI scheme

As performed for the CMOS development, it would be useful to test mechanically each of these structures before any interconnect step. However, at this stage of the process, neither the detection layers, nor the actuation layers are available to allow the dynamic characterization of the mobile structures. As a conclusion, this means that actuation and detection systems external to the device and using nanometer scale probes are still to be proposed.

\section{SUMMARY}

Depending on the level of the technological developments, the characterization techniques are mature to support them or still require protocol definition and relevance demonstration for the issues addressed. For Beyond CMOS and Extreme CMOS devices, the integration of nano-objects like nanowires and carbon nanotubes, brings about analysis requirements that are at the frontier of the state-of-theart characterization techniques. The specific limitations of the use of the existing physical and chemical characterization techniques for integrated nanomaterials have been highlighted from three different characterization aspects.
First, the use of Scanning Probe Microscopy has demonstrated that keeping the intrinsic properties of nanomaterials during their integration is a central issue. Moreover, in-situ localization and positioning are specifically challenging and data analysis is mainly statistical.

Then the extraction of the reliable 3D information that is mandatory for integrated nanomaterials needs specific sample preparation. Nanoneedle sample preparation is demonstrated to overcome some reconstruction artifacts. However, the need for full $360^{\circ}$ tilt series and reconstruction algorithms taking into account physical properties of the integrated materials, like heavy elements, are required for reliable results.

Finally, the measurement developments related to NEMS technologies guided by the need for dynamic characterization of these components are shown to be the key issue for the VLSI objective.

\section{ACKNOWLEDGMENTS}

This work was supported by the French National Research Agency (ANR) through the Carnot funding and the Basic Technological Research Program.

\section{REFERENCES}

1. A. Bailly et al., "Direct quantification of gold along a single Si nanowire”, Nanoletter 8, 11, .3708-14 (2008)

2. M. A. Reed et al., "Conductance of a molecular junction”, Science 278, 252 (1997)

3. V. Petkov et al., "Structure beyond Bragg: Study of $\mathrm{V}_{2} \mathrm{O}_{5}$ nanotubes”, Phys. Rev. B: Condens. Matter 69, 085410 (2004)

4. P. Holliger et al., "SIMS depth profiling of ultrashallow $\mathrm{P}, \mathrm{Ge}$ and As implants in Si using $\mathrm{MCs}_{2}{ }^{+}$ions", Surface and Interface Analysis 34, 472 (2002).

5. S. Lhostis et al., "Crystalline structure of HfZrO thin films and $\mathrm{ZrO}_{2} / \mathrm{HfO}_{2}$ bi-layers grown by AVD for MOS applications”, ECS Trans. 13, 101 (2008)

6. N. Barrett et al., "Core-level photoemission study of nitrided hafnium silicate thin films", Microelectronic Engineering 84, 2302-305 (2007).

7. E. Martinez et al., "Electronic and chemical properties of the TaN/a-SiOC:H stack studied by photoelectron spectroscopy for advanced interconnects”, J. Appl. Phys. 104, 073708 (2008)

8. N. Rochat et al., "Enhancement of infrared spectroscopy capabilities for nanoelectronic and nanotechnology applications”, AIP Conference Proceedings 931, edited by D. G. Seiler et al., American Institute of Physics, Melville, NY, 2007 pp. 79-83

9. J.-P. Barnes et al., “ToF-SIMS imaging of $\mathrm{Cl}$ at $\mathrm{Cu}$ grain boundaries in interconnects for microelectronics", Applied Surface Science 255, 4, 1564-1568 (2007)]

10. O. Renault et al., "Energy-filtered photoelectron emission microscopy (EF-PEEM) for imaging 
nanoelectronic materials", AIP Conference Proceedings 931, edited by D. G. Seiler et al., American Institute of Physics, Melville, NY, 2007 pp. 502-506

11. C. C. Williams et al., "Scanning capacitance microscopy on a 25 nm scale”, Appl. Phys. Lett. 55, 2, 203-5 (1989)

12. H. Edwards et al., "pn-junction delineation in Si devices using scanning capacitance spectroscopy", J. Appl. Phys. 87, 3, 1485-95 (2000)

13. M. Stangoni et al., "Accuracy of scanning capacitance microscopy for the delineation of electrical junctions", $J$. Vac. Sci. Technol. B 22, 406-410 (2004)

14. P. De Wolf et al., "Characterization of a point-contact on silicon using force microscopy-supported resistance measurements", Appl. Phys. Lett. 66, 1530-2 (1995).

15. P. Eyben et al., "Probing semiconductor technology and devices with scanning spreading resistance microscopy"; in: "Scanning Probe Microscopy : Electrical and Electromechanical Phenomena at the Nanoscale", Springer, 31-87 (2007)

16. N. Gaillard et al., "Characterization of electrical and crystallographic properties of metal layers at decananometer scale using Kelvin probe force microscope", Microelectronic Engineering 83, 11-12, 2169-74 (2006)

17. K. van Benthem et al., "Three-dimensional imaging of individual hafnium atoms inside a semiconductor device”, Appl. Phys. Lett. 87, 034104 (2005)

18. D. Cooper et al., "Medium resolution off-axis electron holography with millivolt sensitivity", Appl. Phys. Lett. 91, 143501 (2007)

19. D. Cooper et al., "The ultimate performance of off-axis electron holography in doped specimens prepared using focused ion beam milling", submitted to Phys. Rev. B, (2009)

20. F. Pulizzi, "Electron and x-ray microscopy “, Nature Materials Insight 8, 259-301 (2009)

21. P. Bleuet et al., "Diffraction-tomography: A local structural probe for heterogeneous diluted materials", Nature Materials 7, 468-72 (2008)

22. P. Pareige et al., "Atomic resolution analyses of nanostructured materials by atom probe tomography", International journal of Nanotechnology 5, 592 (2008)

23. W. Vandervorst, "Semiconductor profiling with sub-nm resolution: Challenges and solutions”, Applied Surface Science 255, 4, 805-12 (2007)]

24. A. Bachtold et al., "Scanned probe microscopy of electronic transport in carbon nanotubes", Phys. Rev. Lett. 84, 6083 (2000)

25. L. Fourdrinier et al., "Electrical properties measurements on individual carbon nanofibers by scanning spreading resistance microscopy”, J. Appl. Phys., 104, 114305 (2008)

26. N. Chevalier et al., "Electrical measurements by scanning spreading resistance microscopy: Application to carbon nanofibers and Si nanowires”, this conference, (2009)

27. E. Latu-Romain et al., "Surface-induced p-type conductivity in $\mathrm{ZnO}$ nanowires investigated by scanning probe microscopy", submitted to Nature Materials.

28. M. Troyon and K. Smaali, "Scanning near-field electron beam induced current microscopy: Application to III-V heterostructures and quantum dots”, Appl. Phys. Lett. 90, 212110 (2007)
29. X. Lin et al., "Intrinsic current-voltage properties of nanowires with four-probe scanning tunneling microscopy: A conductance transition of $\mathrm{ZnO}$ nanowire”, Appl. Phys. Lett. 89, 043103 (2006)

30. M. Shimada et al.,"3-Dimensional structures of pores in low-k films observed by quantitative TEM tomograph and their impact on penetration phenomena”, in Proc. of International Interconnect Technology Conf., 2004, (IEEE, 2004)pp. 178-80

31. A. Yurtsever et al., "Three-dimensional imaging of nonspherical silicon nanoparticles embedded in silicon oxide by plasmon tomography", Appl. Phys. Lett. 89, 151920 (2006)

32. C. Dupre et al, "3D nanowire gate-all-around transistors: Specific integration and electrical features”, Solid State Electronics 52, 4, 519-25 (2008)

33. N. Kawase et al., "Transmission electron microtomography without the "missing wedge" for quantitative structural analysis”, Ultramicroscopy 107, 1, 8-15 (2007),

34. F. Lorut and D. Delille, "Advanced FIB-based sample preparation for 3D characterization of $45 \mathrm{~nm}$ IC's", Extended abstract of a paper presented at Microscopy and Microanalysis 2008 in Albuquerque, New Mexico, USA, August 3 - August 7, 2008

35. S. Bals et al., "Discrete tomography in materials science: less is more?”, in EMC 2008: Instrumentation and Methods 1, edited by M. Luysberg, K. Tillmann and T. Weirich, Berlin Heidelberg: Springer-Verlag, 2008, pp. 291-292

36. T. C. Leondes, "MEMS/NEMS handbook. Techniques and applications", Springer, 2006.

37. A. Bosseboeuf and S. Petitgrand, "Characterization of the static and dynamic behaviour of M(O)EMS by optical techniques : status and trends", Journal of Micromechanics and Microengineering 13, S23-33 (2003)

38. S. Petitgrand and A. Bosseboeuf, "Simultaneous mapping of out-of-plane and in-plane vibrations of MEMS with (sub)nanometer resolution”, Journal of Micromechanics and Microengineering 14, S97-101 (2003)

39. M. Zalalutdinov et al., Frequency-tunable micromechanical oscillator, Appl. Phys. Lett. 77, 3287 (2000) 


\section{CONTENTS}

Preface

\section{TECHNOLOGY OVERVIEW FOR NANOELECTRONICS AND METROLOGY}

Metrology for Emerging Materials, Devices, and Structures: The Example of Graphene $\ldots \ldots \ldots \ldots$ A. C. Diebold

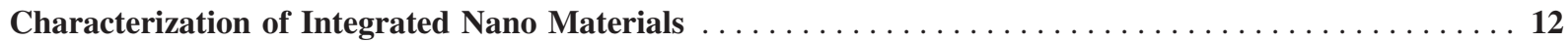
A. Chabli, P. Cherns, N. Chevalier, D. Cooper, D. Lafond, F. Bertin, H. Blanc, A. Brenac, P. Andreucci, and J.-C. Gabriel

\section{METROLOGY FOR CMOS EXTENSION}

Characterization of Organic Contamination in Semiconductor Manufacturing Processes . . . . . . . 23 A. Nutsch, B. Beckhoff, G. Bedana, G. Borionetti, D. Codegoni, S. Grasso, G. Guerinoni, A. Leibold, M. Müller, M. Otto, L. Pfitzner, M.-L. Polignano, D. De Simone, and L. Frey

GIXRF in the Soft X-ray Range Used for the Characterization of Ultra Shallow Junctions . . . . . . . 29 B. Beckhoff, P. Hoenicke, D. Giubertoni, G. Pepponi, and M. Bersani

Ultra-thin AIOx and LaOx Metrology_WD-XRF Technique Development $\ldots \ldots \ldots \ldots \ldots$ C. C. Wang, R. Wang, D. Liu, Y. Cao, X. Tang, Y. Uritsky, and S. Gandikota

On the Use of Synchrotron Radiation for the Characterization of "Tin/HfO," Gate Stacks . . . . . . 40 C. Gaumer, E. Martinez, S. Lhostis, F. Fillot, P. Gergaud, B. Detlefs, J. Roy, Y. Mi, J.-P. Barnes, J. Zegenhagen, and A. Chabli

Multi-technique Characterization of Arsenic Ultra Shallow Junctions in Silicon within the ANNA

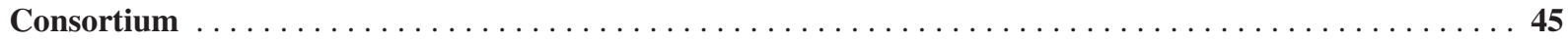
D. Giubertoni, G. Pepponi, B. Beckhoff, P. Hoenicke, S. Gennaro, F. Meirer, D. Ingerle, G. Steinhauser, M. Fried, P. Petrik, A. Parisini, M. A. Reading, C. Streli, J. A. van den Berg, and M. Bersani

NIST High Resolution X-ray Diffraction Standard Reference Material: SRM $2000 \ldots \ldots \ldots \ldots$ D. Windover, D. L. Gil, A. Henins, and J. P. Cline

Characterization of $\mathrm{HfO}_{2}$ and Hafnium Silicate Films on $\mathrm{SiO}_{2} / \mathrm{Si} \ldots \ldots \ldots \ldots \ldots \ldots \ldots$ E. Bersch, M. Di, S. Consiglio, R. D. Clark, G. J. Leusink, and A. C. Diebold

Non-traditional Spectroscopy for Analysis of Semiconductor and Photovoltaic Thin Films Materials . . 62 F. Li and S. Anderson

Detection of Metal Contamination on Silicon Wafer Backside and Edge by New TXRF Methods . . . . 67 H. Kohno, M. Yamagami, J. Formica, and L. Shen

Vacuum-Ultraviolet Reflectometry of Ultra-thin $\mathbf{H f O}_{\mathbf{2}}$ Films $\ldots \ldots \ldots \ldots \ldots \ldots \ldots \ldots \ldots \ldots \ldots \ldots$ J. Hurst and V. Vartanian

Application of the SPV-based Surface Lifetime Technique to In-line Monitoring of Surface $\mathrm{Cu}$

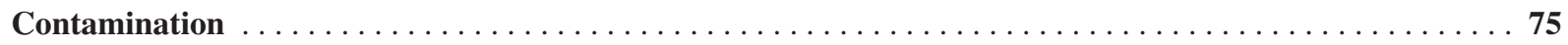
J. D'Amico, A. Savtchouk, M. Wilson, C. H. Kim, H. W. Yoo, C. H. Lee, T. K. Kim, and S. H. Son

High-resolution Rutherford Backscattering Analysis of Nanoscale Thin Films $\ldots \ldots \ldots \ldots$ J. D. LaRose, M. Huang, E. Bersch, M. Di, A. C. Diebold, S. Consiglio, R. D. Clark, and G. J. Leusink

Evaluation of Experimental Techniques for In-line Ion Implantation Characterization $\ldots \ldots \ldots \ldots . .84$ L. Vignoud, F. Milesi, E. Nolot, A. Danel, and S. Favier

Advanced Gate and Stack Dielectric Characterization with FastGate ${ }^{\circledR}$ Technology $\ldots \ldots \ldots . \ldots .{ }^{2}$ R. J. Hillard, L. C. Tan, and K. G. Reid

Towards Routine Backside SIMS Sample Preparation for Efficient Support of Advanced IC

Process Development $\ldots \ldots \ldots \ldots \ldots \ldots \ldots \ldots \ldots \ldots \ldots \ldots \ldots \ldots \ldots \ldots \ldots \ldots \ldots \ldots \ldots \ldots \ldots$ M. J. P. Hopstaken, C. Cabral Jr., D. Pfeiffer, C. Molella, and P. Ronsheim

X-ray Photoelectron Spectromicroscopy of Doped Silicon Patterns N. Barrett, M. Lavayssière, L. F. Zagonel, A. Bailly, and O. Renault 
Spectroscopic Ellipsometry Characterization of High-k Films on $\mathbf{S i O}_{2} / \mathbf{S i} \ldots \ldots \ldots \ldots \ldots \ldots \ldots$ M. Di, E. Bersch, S. Consiglio, T. Zhang, P. Tyagi, R. D. Clark, G. J. Leusink, A. Srivatsa, and A. C. Diebold

Photoreflectance Spectroscopic Characterizations of $\mathrm{Si}$ with $\mathrm{SiO}_{2}$ and $\mathrm{HfO}_{2}$ Dielectric Layers ...... 109 T. Zhang, M. Di, E. J. Bersch, H. Chouaib, A. Salnik, L. Nicolaides, C. Bevis, S. Consiglio, R. D. Clark, and A. C. Diebold

Thickness Measurement of Thin-metal Films by Optical Metrology $\ldots \ldots \ldots \ldots \ldots \ldots \ldots \ldots \ldots \ldots$ V. K. Kamineni, M. Raymond, E. J. Bersch, B. B. Doris, and A. C. Diebold

Post-deposition Annealing Analysis for $\mathrm{HfO}_{2}$ Thin Films Using GIXRR/GIXRD 122 W.-E. Fu, Y.-Q. Chang, and Y.-C. Chen

\section{METROLOGY FOR EXTREME CMOS DEVICES}

Scaling Effects on Ferro-Electrics: Application in Nanoelectronics and Characterization

B. Vilquin, B. Gautier, A. Brugère, and J. S. Moulet

Molecular Dynamics Calculation of Thermal Conductivity of Graphene Nanoribbons 135

J. Hu, X. Ruan, Z. Jiang, and Y. P. Chen

Characterization of Nano-Scale Graphene Devices for Thickness and Defect Metrology Using

Micro- and Nano-Raman Spectroscopy

G. Rao, S. McTaggart, J. U. Lee, and R. E. Geer

\section{INTERCONNECTS}

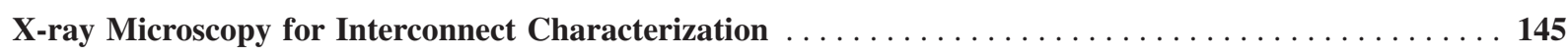

J. Gelb, W. Yun, M. Feser, and A. Tkachuk

Porous SiOCH Post Plasma Damage Characterization Using Ellipsometric Porosimetry . . . . . . . . 149

C. Licitra, R. Bouyssou, T. Chevolleau, N. Rochat, and F. Bertin

EBSD Analysis of Narrow Damascene Copper Lines ............................. 154

R. H. Geiss, D. T. Read, G. B. Alers, and R. L. Graham

Scanning Acoustic Microscopy of 3D-Interconnect . . . . . . . . . . . . . . . . . . . . . . . . . 159

L.-W. Kong, A. C. Diebold, A. Rudack, and S. Arkalgud

X-ray Scattering Methods for Porosity Metrology of Low- $\kappa$ Thin Films $\ldots \ldots \ldots \ldots \ldots \ldots \ldots \ldots$

C. M. Settens, V. K. Kamineni, G. A. Antonelli, A. Grill, A. C. Diebold, and R. J. Matyi

Spectroscopic Ellipsometry of Porous Low- $\boldsymbol{\kappa}$ Dielectric Thin Films $\ldots \ldots \ldots \ldots \ldots \ldots \ldots \ldots \ldots$

V. Kamineni, C. M. Settens, A. Grill, G. A. Antonelli, R. J. Matyi, and A. C. Diebold

\section{CHARACTERIZATION METHODS}

Modeling Artifacts in the Analysis of Test Semiconductor Structures in Atom Probe Tomography . . . 175

F. Vurpillot, M. Gruber, S. Duguay, E. Cadel, and B. Deconihout

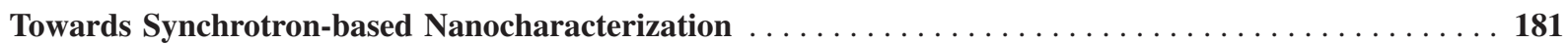

P. Bleuet, L. Arnaud, X. Biquard, P. Cloetens, L. Doyen, P. Gergaud, P. Lamontagne, M. Lavayssière, J.-S. Micha,

O. Renault, F. Rieutord, J. Susini, and O. Ulrich

Wave Front Sensor for Highly Accurate Characterization of Flatness on Wafer Surfaces . . . . . . . 188

A. Nutsch, S. Bucourt, T. Grandin, I. Lazareva, and L. Pfitzner

Nanoscale Characterization of Ultra-thin Dielectrics Using Scanning Capacitance Microscopy . . . . 193

O. Ligor, B. Gautier, A. Descamps, D. Albertini, L. Militaru, and N. Baboux

Reference-free Characterization of Semiconductor Surface Contamination and Nanolayers by

X-ray Spectrometry . . . . . . . . . . . . . . . . . . . . . . . . . . . . . . . . . 198

B. Beckhoff, R. Fliegauf, P. Hönicke, M. Kolbe, M. Müller, B. Pollakowski, F. Reinhardt, J. Weser, and G. Ulm

Polarized Optical Scattering Measurements of Metallic Nanoparticles on a Thin Film Silicon Wafer . . 203

C.-Y. Liu, T.-A. Liu, and W.-E. Fu

Temperature-programmed Gas-Sensing with Microhotplates: An Opportunity to Enhance

Microelectronic Gas Sensor Metrology

J. Geist and M. Afridi 
Enhanced Spatial Resolution Scanning Kelvin Force Microscopy Using Conductive Carbon

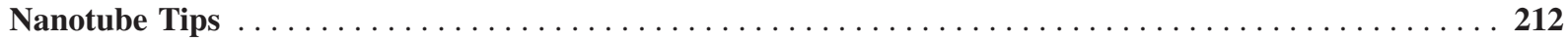

J. J. Kopanski, P. McClure, and V. Mancevski

Application of Micro-thermal Analysis for Metal, Oxide, and Non-oxide Thin Film Materials

N. Carlie, J. Massera, L. Petit, and K. Richardson

Effects of Experimental Parameters on the Work Function Measurement: A Kelvin Force Microscopy

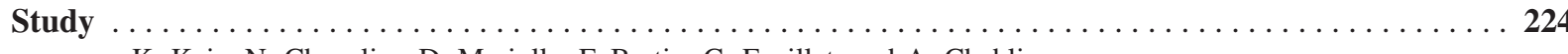

K. Kaja, N. Chevalier, D. Mariolle, F. Bertin, G. Feuillet, and A. Chabli

\section{MICROSCOPY FOR NANOELECTRONICS}

Aberration-corrected Electron Microscopy Imaging for Nanoelectronics Applications $\ldots \ldots \ldots \ldots \ldots 231$

C. Kisielowski, P. Specht, D. Alloyeau, R. Erni, and Q. Ramasse

Corrected Electron Optics_-Improved Resolution and New Analysis Capabilities . . . . . . . . . 241

M. Steigerwald, G. Benner, U. Mantz, and D. Preikszas

Understanding Imaging and Metrology with the Helium Ion Microscope $\ldots \ldots \ldots \ldots \ldots \ldots \ldots$

M. T. Postek, A. E. Vladár, and B. Ming

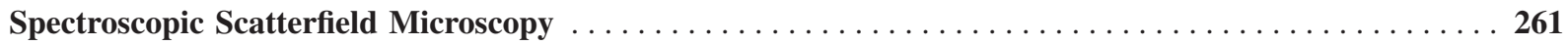

B. M. Barnes, N. A. Heckert, R. Quintanilha, H. Zhou, and R. M. Silver

Understanding the Phase Images from Off-Axis Electron Holography $\ldots \ldots \ldots \ldots \ldots \ldots$

D. Cooper, J.-M. Hartmann, J.-P. Barnes, and A. Chabli

Simulation Study of Transmission Electron Microscopy Imaging of Graphene Stacking $\ldots \ldots \ldots \ldots 271$ F. Nelson, A. C. Diebold, and R. Hull

Enhanced TEM Sample Preparation Using in-situ Low Energy Argon Ion Milling $\ldots \ldots \ldots \ldots \ldots$

H. Stegmann, Y. Ritz, D. Utess, R. Hübner, and E. Zschech

Correction of Hysteresis in SPM Images by a Moving Window Correlation Method $\ldots \ldots \ldots \ldots$

J. Fu, W. Chu, R. Dixson, G. Orji, and T. Vorburger

Electrical Measurements by Scanning Spreading Resistance Microscopy: Application to Carbon

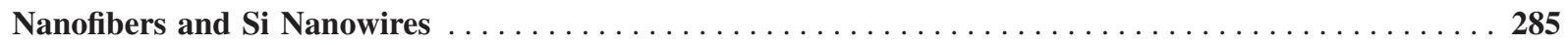

N. Chevalier, D. Mariolle, L. Fourdrinier, C. Celle, C. Mouchet, S. Poncet, J. P. Simonato, H. Le Poche,

E. Rouviere, F. Bertin, and A. Chabli

A Study of Gate-All-Around Transistors by Electron Tomography $\ldots \ldots \ldots \ldots \ldots \ldots \ldots \ldots$

P. D. Cherns, F. Lorut, S. Beçu, C. Dupré, K. Tachi, D. Cooper, A. Chabli, and T. Ernst

Quantifying and Enforcing the Two-dimensional Symmetries in Scanning Probe Microscopy Images . . 294 P. Moeck, M. Toader, M. Abdel-Hafiez, and M. Hietschold

Precession Electron Diffraction and Its Utility for Structural Fingerprinting in the Transmission

Electron Microscope . . . . . . . . . . . . . . . . . . . . . . . . . . . . . . . 299

P. Moeck, S. Rouvimov, and S. Nicolopoulos

Automated Crystal Phase and Orientation Mapping of Nanocrystals in a Transmission Electron

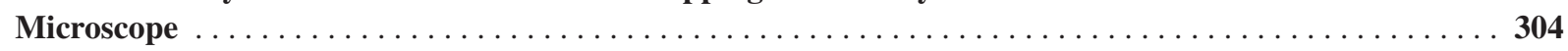

P. Moeck, S. Rouvimov, E. F. Rauch, and S. Nicolopoulos

Helium Ion Beam Microscopy for Copper Grain Identification in BEOL Structures . . . . . . . . . 309

R. J. J. van den Boom, H. Parvaneh, D. Voci, C. Huynh, L. Stern, K. A. Dunn, and E. Lifshin

\section{ELECTRICAL MEASUREMENTS AT NANO DIMENSIONS}

Boron Nanowires for Flexible Electronics and Field Emission $\ldots \ldots \ldots \ldots \ldots \ldots \ldots \ldots \ldots$

J. Tian, J. Cai, C. Hui, C. Li, Y. Tian, C. Shen, and H. Gao

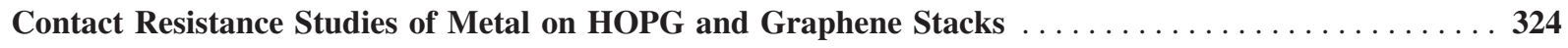

A. Venugopal, A. Pirkle, R. M. Wallace, L. Colombo, and E. M. Vogel

Advanced Capacitance Metrology for Nanoelectronic Device Characterization $\ldots \ldots \ldots \ldots \ldots$

C. A. Richter, J. J. Kopanski, C. Jiang, Y. Wang, M. Y. Afridi, X. Zhu, D. E. Ioannou, and Q. Li

In-line $90 \mathrm{~nm}$ Technology Gate Oxide Nitrogen Monitoring with Non-contact Electrical Technique . . 333

N. Pic, G. Polisski, E. Paire, V. Rizzo, C. Grosjean, B. Bortolotti, J. D’Amico, and N. Cabuil

The Effect of Surface Conditioning on Silicon Wafer Resistivity Monitoring $\ldots \ldots \ldots \ldots 38$

E. Tsidilkovski and A. Bertuch 
Variable Temperature Measurements in Cryogenic Probe Stations

\section{METROLOGY FOR PATTERNING}

Integrated ODP Metrology Matching to Reference Metrology for Lithography Process Control . . . . 349

P. Kearney, J. Uchida, H. Weichert, D. Likhachev, D. Hetzer, and G. Fleischer

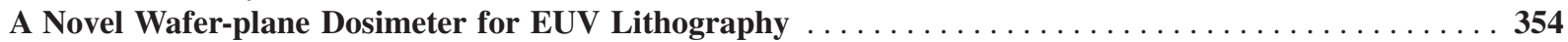

S. Grantham and C. Tarrio

Interference Microscopy for Semiconductor Back End Patterning Metrology . . . . . . . . . . 359

X. Colonna de Lega, M. Fay, R. Kruse, D. Grigg, M. Darwin, M. Knowles, J. Barnak, and M. Wu

Compact X-ray Tool for Critical-dimension Metrology $\ldots \ldots \ldots \ldots \ldots \ldots \ldots \ldots \ldots \ldots \ldots \ldots \ldots$

B. Yokhin, A. Krokhmal, A. Dikopoltsev, D. Berman, I. Mazor, B.-H. Lee, D.-C. Ihm, and K. H. Kim

THEORY, MODELING, AND SIMULATION

Simulations of Scatterometry Down to $22 \mathrm{~nm}$ Structure Sizes and beyond with Special Emphasis

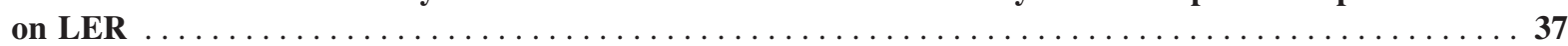

W. Osten, V. Ferreras Paz, K. Frenner, T. Schuster, and H. Bloess

Spectroscopic Polarimetry of Light Scattered by Surface Roughness and Textured Films in

Nanotechnologies

F. Ferrieu

Application of Statistical Dynamical X-ray Diffraction Theory to Defective Semiconductor

Heterostructures

P. K. Shreeman and R. J. Matyi

Author Index

Key Word Index

395 